SHORT REPORT

\title{
Assessment of the impact of the removal of cerebrospinal fluid on cerebral tissue volumes by advanced volumetric 3D-MRI in posthaemorrhagic hydrocephalus in a premature infant
}

\author{
R W Hunt, S K Warfield, H Wang, M Kean, J J Volpe, T E Inder
}

J Neurol Neurosurg Psychiatry 2003;74:658-660

Current clinical practice in the premature infant with posthaemorrhagic ventricular dilatation (PHVD) includes drainage of cerebrospinal fluid (CSF). This case study used advanced volumetric three dimensional magnetic resonance imaging to document the impact of CSF removal on the volume of regional brain tissues in a premature infant with PHVD. The removal of a large volume of CSF was associated with an identical reduction in CSF volume, but more dramatically with a significant increase in the regional volumes of cortical grey matter and myelinated white matter. The alterations in cerebral cortical grey matter and myelinated white matter volumes may provide insight into the established association of PHVD with deficits in cognitive and motor functions.

$\mathrm{P}$ rogressive posthaemorrhagic ventricular dilatation (PHVD) remains a significant problem in the premature infant. PHVD occurs in approximately $25 \%$ of premature infants with intraventricular haemorrhage ${ }^{1}$ and the morbidity of the condition is appreciable. The primary mechanisms responsible for the PHVD include impaired cerebrospinal fluid (CSF) absorption resulting in the acute phase from particulate blood clot and in the chronic phase from a secondary obliterative arachnoiditis. ${ }^{2}$ Experimental studies indicate that hydrocephalus has deleterious effects on cerebral white matter, initially involving oligodendrocytes and later axons. ${ }^{3-5}$ The mechanisms appear to be related to attenuation of vessels in white matter, with ischaemia and altered energy metabolism. ${ }^{4-7}$ Studies in human infants with PHVD also suggest ischaemia, alterations in myelin and axons in white matter, and physiological disturbances (impaired visual and somatosensory evoked potentials), consistent with cerebral white matter injury. ${ }^{24-10}$ Notably, however, experimental studies have also shown deleterious effects on cerebral cortical neurones, ${ }^{3811}$ and findings on follow up of human infants include cognitive deficits, supporting an effect on cerebral cortical neuronal effects. ${ }^{90}$

The serial removal of aliquots of CSF in premature infants with PHVD has been shown to have beneficial effects on cerebral perfusion and metabolism. ${ }^{2}{ }^{12}$ Cranial ultrasound scans done immediately after CSF removal have also shown a decrease in ventricular size. ${ }^{213}$ However, the quantitative aspects of this decrease are difficult to delineate by ultrasonography, and more importantly it has not been possible to determine the quantitative effects of removal of CSF on other regional brain structures by this imaging method.

We report a case in which we investigated the changes in regional brain volume using three dimensional magnetic resonance imaging (3d-MRI) after removal of CSF in an infant with PHVD.

\section{CASE REPORT}

A female infant (twin II) was born at 28 weeks' gestation (birth weight $1300 \mathrm{~g}$ ) to an otherwise healthy primigravid woman with a known twin pregnancy. Her pregnancy was complicated by spontaneous rupture of the first twin's amniotic sac at 15 weeks and by an antepartum haemorrhage with spontaneous onset of labour at 28 weeks' gestation. The mother had received a full course of antenatal steroids before delivery. The twins were delivered by emergency caesarean section. Apgar scores for this infant were 6 and 9 at one and five minutes, respectively. The infant's initial course was complicated by hyaline membrane disease requiring three days of mechanical ventilation and an additional two days of continuous positive airway pressure (CPAP). A small pneumothorax and pneumomediastinum were noted on day 2 and resolved with conservative management.

On day 1 of life, cranial ultrasound scan revealed bilateral parieto-occipital echo densities. On day 3 there was bilateral intraventricular haemorrhage, with clot extending to the third and fourth ventricles, and a right frontal parenchymal echo density consistent with parenchymal haemorrhagic venous infarction. By day 8 there was sonographic evidence of early ventricular dilatation which had progressed by day 21 . By day 28 there was marked ventriculomegaly with right frontal cystic periventricular leucomalacia. At that time head circumference was increasing by $2 \mathrm{~cm}$ a week but the infant was clinically stable.

A right frontal Rickham reservoir was inserted on day 28 because of the progressive ventricular dilatation and rapid head growth. CSF was removed daily for three days after insertion $(10 \mathrm{ml} / \mathrm{kg} /$ day $)$. Because the infant was enrolled in a research magnetic resonance imaging (MRI) protocol for an initial scan at 30-32 weeks, MRI was done on day 31. Because of the degree of ventricular dilatation, three taps of the reservoir were made, with removal of volumes of $18 \mathrm{ml}(10 \mathrm{ml} / \mathrm{kg})$, $27 \mathrm{ml}(15 \mathrm{ml} / \mathrm{kg})$, and $27 \mathrm{ml} \mathrm{(15} \mathrm{ml/kg),} \mathrm{two} \mathrm{hours} \mathrm{apart} \mathrm{over}$ a period of six hours. Multiple taps were undertaken because the fontanel remained full after the initial two taps. MRI was done again after the three reservoir taps. All scans were obtained with a 1.5 Tesla General Electric Signa System (GEMedical Systems, Milwaukee, Wisconsin, USA). For the acquisition of the primary magnetic resonance data two different imaging modes were applied: a three dimensional (3D) Fourier transform spoiled gradient recalled (SPGR) sequence ( $1.5 \mathrm{~mm}$ coronal slices; flip angle $45^{\circ}$; repetition time $35 \mathrm{~ms}$; echo time $5 \mathrm{~ms}$; field of view $18 \mathrm{~cm}$; matrix $256 \times 256)$, and a coronal T2 weighted sequence ( $1.7 \mathrm{~mm}$ coronal slices; repetition time $3000 \mathrm{~ms}$; echo times $162 \mathrm{~ms}$; field of view $18 \mathrm{~cm}$; matrix $256 \times 256$ ). 

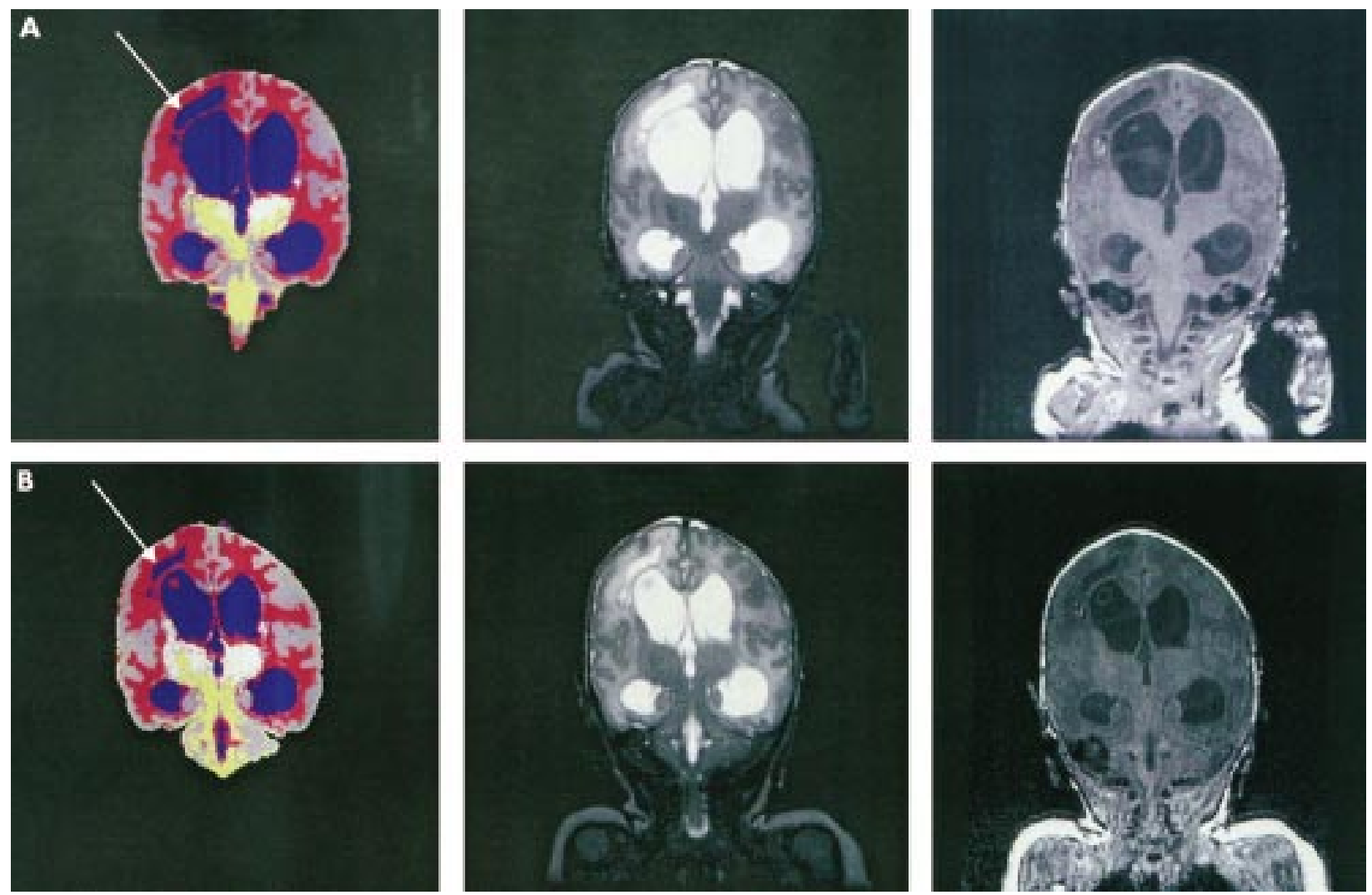

Figure 1 Regional segmentation maps (left panels), coronal T2 weighted images (middle panels), and coronal T1 weighted images (right panels) before (A) and after (B) the removal of CSF from the Rickham's reservoir. Regional segmentation maps show cerebrospinal fluid (blue), unmyelinated white matter (red), myelinated white matter (yellow), basal ganglia (white), and cortical grey matter (grey). The images show the cystic right frontal parenchymal lesion (arrow). There is only a small apparent reduction qualitatively in the CSF volume following the CSF removal on the conventional images; this reduction is more apparent with the segmented maps.

Postacquistion processing was carried out on workstations (Sun Microsystems, Mountain View, California, USA) with newly developed software. A sequence of image processing algorithms was used to segment each of the MRI slices into separate tissue classes: cortical grey matter, subcortical grey matter, unmyelinated white matter, myelinated white matter, and CSF. ${ }^{14-16}$ These algorithms were designed to reduce imaging system noise, identify a linear transformation to align the DE spin echo images with the SPGR images to form a three channel dataset, resample the DE spin echo images according to this transform, classify tissue types on the basis of the MR intensity in the three channels, and identify tissue class surfaces for three dimensional visualisation.

These analyses were done on the MRI studies performed before and after the taps of the reservoir. The segmented images and conventional coronal MRI images before and after the removal of a total of $72 \mathrm{ml} \mathrm{CSF}$ are shown in fig 1 .

The cortical grey matter, CSF, myelinated and unmyelinated white matter, basal ganglia, and total intracranial volumes were calculated (table 1). The removal of CSF resulted in a decrease of $71.9 \mathrm{ml} \mathrm{CSF} \mathrm{(34 \%} \mathrm{of} \mathrm{the} \mathrm{pre-tap} \mathrm{value),} \mathrm{a} \mathrm{decrease}$ almost identical to the volume of $72 \mathrm{ml}$ removed over the six hours between the MRI scans. The largest change in regional brain volume involved the cerebral cortical grey matter, the absolute volume of which increased by $44.4 \mathrm{ml}$ (39\% more than the pre-tap volume). There also was an increase in the absolute volume of myelinated white matter $(6.5 \mathrm{ml}$ or $61 \%$ more than the pre-drainage volume). However, because the absolute volume of cerebral myelinated white matter constitutes such a small proportion of total brain volume at this stage of maturation, this increase in volume was only $15 \%$ of the $44.4 \mathrm{ml}$ increase in cerebral cortical grey matter volume.
Table 1 Intracranial cerebral tissue volumes $(\mathrm{ml})$ before and after removal of cerebrospinal fluid $(72 \mathrm{ml})$ from a Rickham reservoir in a premature infant with PHVD

\begin{tabular}{lll}
\hline Intracranial volumes & $\begin{array}{l}\text { Before removal of } \\
\text { CSF }\end{array}$ & $\begin{array}{l}\text { After removal of } \\
\text { CSF }\end{array}$ \\
\hline CSF & 211.1 & 139.2 \\
Cortical grey matter & 112.8 & 157.2 \\
Myelinated white matter & 10.7 & 17.2 \\
Unmyelinated white matter & 199.9 & 185.5 \\
Basal ganglia & 5.4 & 6.3 \\
Total Intracranial volume & 539.9 & 505.4 \\
\hline
\end{tabular}

\section{DISCUSSION}

This case report is the first to delineate clearly in vivo the impact of CSF removal on intracranial tissue volumes in a premature infant with PHVD. With advanced volumetric 3D-MRI techniques we showed that the removal of $72 \mathrm{ml}$ of CSF resulted in an identical reduction of intracranial CSF volume $(71.9 \mathrm{ml})$. However, more strikingly the removal of this CSF volume was associated with a pronounced increase in the volumes of cortical grey matter (39\%) and myelinated white matter $(61 \%)$. In contrast, there was no definite alteration in the unmyelinated white matter or basal ganglia volumes. The data may provide insight into the impact of PHVD in the premature infant on cerebral development.

The most striking increase in tissue volume after CSF removal involved cerebral cortical grey matter. Experimental 
studies of hydrocephalus have shown morphological and biochemical disturbances in the cerebral cortex, including alterations in synaptogenesis and evidence for neuronal degeneration. ${ }^{8}{ }^{17}$ In the human brain between 32 weeks postconceptional age (the age of our infant) and 40 weeks, the cerebral cortical grey matter volume increases threefold. ${ }^{15}$ The likely anatomical correlates of this change include elaboration of dendritic and axonal ramifications and synaptogenesis. ${ }^{2}$

The ventricular distension associated with PHVD may impair cerebral cortical grey matter development in at least two ways. Axonal destruction in PHVD might result in input deprivation and output isolation of the overlying grey matter and hence impaired cerebral cortical neuronal differentiation. ${ }^{18}$ However, even without overt axonal destruction, the presence of the ventricular distension alone might alter the "tension" applied to the developing cortex by descending fibres in the white matter. Theoretical modelling and experimental observations suggest that fibre development in cerebral white matter is crucial for gyral development. ${ }^{19}$ With PHVD such "tension based morphogenesis" might be impaired and thereby alter cerebral cortical development. However regardless of the mechanism, the alteration in cerebral cortical grey matter volume documented in this study may provide insight into the established association of PHVD with deficits in cognitive function. ${ }^{10}$ The dramatic increase in cortical grey matter volume following drainage of the CSF may also provide a correlate for understanding the potential benefit from early ventricular drainage on long term neurodevelopmental outcome.

A prominent increase in the volume of myelinated white matter was also documented following CSF drainage. In experimental and human studies of PHVD, a reduction in myelin associated enzymes and structural proteins, with subsequent impaired myelination and axonal loss, has been shown in the cerebral white matter. ${ }^{20}$ These disturbances in myelination and axonal pathways may be the correlates for the increased risk of adverse motor outcome in PHVD. ${ }^{90}$ Evidence from the hydrocephalic animal model shows clearly that early drainage of dilating ventricles at one week but not at four weeks, when axonal loss had occurred, allowed recovery of myelination. ${ }^{20}$ Our findings are consistent with the suggestion from follow up studies that early adequate drainage may allow the normal developmental sequence of myelination and improve long term outcome. ${ }^{9}$

\section{Conclusions}

Our findings show the utility and accuracy of volumetric 3D-MRI in defining the impact of the removal of CSF on intracranial regional tissue volumes, with dramatic alterations in cortical grey matter and myelinated white matter following CSF removal. These findings also support the benefit of removing CSF in PHVD, but require extended validation with a larger population of infants including correlation with later neurodevelopmental outcome.

\section{ACKNOWLEDGEMENTS}

This investigation was supported in part by NIH grant P41 RR13218 and the Whittaker Foundation (USA), and by the Jack Brockhoff Foundation (Australia).

\section{Authors' affiliations}

R W Hunt, H Wang, T E Inder, Department of Neonatal Neurology, Royal Children's Hospital, University of Melbourne, Australia

M Kean, Department of Medical Imaging, Royal Children's Hospital

J J Volpe, Department of Neurology, Harvard Medical School, Boston, Massachusetts, USA

Competing interests: none declared

Correspondence to: Dr Terrie Inder, Department of Neonatal Neurology, Level 2, Royal Children's Hospital, Flemington Road, Parkville, VIC 3052 Australia; indert@cryptic.rch.unimelb.edu.au

Received 10 September 2002

In revised form 26 November 2002

Accepted 11 January 2003

\section{REFERENCES}

1 Murphy BP, Inder TE, Rooks V, et al. Posthemorrhagic ventricular dilatation in the premature infant - natural history and predictors of outcome. Arch Dis Child 2002;87:F37-41.

2 Volpe JJ. Neurology of the newborn, 4th ed. Philadelphia: WB Saunders, 2001.

3 Wright LC, McAllister JP, Katz SD, et al. Cytological and cytoarchitectural changes in the feline cerebral cortex during experimental infantile hydrocephalus. Pediatr Neurosurg 1990;16:139-55

4 Wozniak M, McLone DG, Raimondi AJ. Micro- and macrovascular changes as the direct cause of parenchymal destruction in congenital murine hydrocephalus. J Neurosurg 1975;43:535-45

5 Jones HC, Richards JK, Bucknall RM, et al. Local cerebral blood flow in rats with congenital hydrocephalus. J Cereb Blood Flow Metab rats with congenital

6 Braun KP, Dijkhuizen RM, deGraaf RA, et al. Cerebral ischemia and white matter edema in experimental hydrocephalus: a combined in vivo MRI and MRS study. Brain Res 1997;757:295-8.

7 Soul JS, Taylor GA, Wypij D, et al. Noninvasive detection of changes in cerebral blood flow by near-infrared spectroscopy in a piglet model of hydrocephalus. Pediatr Res 2000;48:445-9.

8 Boillat CA, Jones JC, Kaiser GL, et al. Ultrastructural changes in the deep cortical pyramidal cells of infant rats with inherited hydrocephalus and the effect of shunt treatment. Exp Neurol 1997;147:377-88.

9 deVries LS, Liem KD, vanDijk K, et al. Early versus late treatment of posthaemorrhagic ventricular dilatation: results of a retrospective study from five neonatal intensive care units in The Netherlands. Acta Paediatr 2002;91:212-17.

10 Dykes FD, Dunbar B, Lazarra A, et al. Posthemorrhagic hydrocephalus in high-risk preterm infants; natural history, management, and long-term outcome. J Pediatr 1989;114:611-18.

11 Miyazawa T, Sato K. Learning disability and impairment of synaptogenesis in HTX-rats with arrested shunt-dependent hydrocephalus. Childs Nerv Sys 1991:7:121-8.

12 Taylor GA, Madsen JR. Neonatal hydrocephalus: hemodynamic response to fontanelle compression - correlation with intracranial pressure and need for shunt placement. Radiology 1996;201:685-9.

13 Rennie JM. Neonatal cerebral ultrasound. Cambridge: Cambridge University Press, 1997

14 Warfield SK, Kaus M, Jolesz FA, et al. Adaptive, template moderated, spatially varying statistical classification. Med Image Anal 2000;4:43-55.

15 Huppi PS, Warfield S, Kikinis R, et al. Quantitative magnetic resonance imaging of brain development in premature and mature newborns. Ann Neurol 1998;43:224-35

16 Inder TE, Huppi PS, Warfield S, et al. Periventricular white matter injury in the premature infant is followed by reduced cerebral cortical grey matter volume at term. Ann Neurol 1999:46:755-60.

17 Suda K, Sato K, Takeda N, et al. Early ventriculoperitoneal shunt effects on learning ability and synaptogenesis of the brain in congenitally hydrocephalic HTX rats. Childs Nerv Sys 1994;10:19-23.

18 Marin-Padilla $M$. Developmental neuropathology and impact of perinatal brain damage. 2. White matter lesions of the neocortex J Neuropathol Exp Neurol 1997:56:219-35.

19 Van Essen DC. A tension-based theory of morphogenesis and compact wiring in the central nervous system. Nature 1997;385:313-18.

20 Del Bigio MR, Kanfer JN, Zhang YW. Myelination delay in the cerebral white matter of immature rats with kaolin-induced hydrocephalus is reversible. J Neuropathol Exp Neurol 1997;56:1053-66. 